\title{
A Delay-Dependent LMI Approach to Dynamics Analysis of Discrete-Time Recurrent Neural Networks with Time-Varying Delays
}

\author{
Qiankun Song ${ }^{a}$ and Zidong Wang,*
}

\begin{abstract}
In this Letter, the analysis problem for the existence and stability of periodic solutions is investigated for a class of general discrete-time recurrent neural networks with time-varying delays. For the neural networks under study, a generalized activation function is considered, and the traditional assumptions on the boundedness, monotony and differentiability of the activation functions are removed. By employing the latest free-weighting matrix method, an appropriate Lyapunov-Krasovskii functional is constructed and several sufficient conditions are established to ensure the existence, uniqueness, and globally exponential stability of the periodic solution for the addressed neural network. The conditions are dependent on both the lower bound and upper bound of the time-varying time delays. Furthermore, the conditions are expressed in terms of the linear matrix inequalities (LMIs), which can be checked numerically using the effective LMI toolbox in MATLAB. Two simulation examples are given to show the effectiveness and less conservatism of the proposed criteria.
\end{abstract}

\section{Keywords}

Discrete-time recurrent neural network; Time-varying delays; Periodic solution; Exponential stability; LyapunovKrasovskil functional; Linear matrix inequality

\section{INTRODUCTION}

In the past few decades, delayed recurrent neural networks (especially delayed Hopfield neural networks, delayed cellular neural networks and delayed bidirectional associative memory neural networks) have found successful applications in many areas such as signal processing, pattern recognition, associative memories, and optimization solvers. Many important results have been reported on the existence, uniqueness, and global asymptotic or exponential stability of the equilibrium point for recurrent neural networks with constant delays or time-varying delays, see $[1,2,7,8,10,17,18,22-25]$ and the references therein for some recent publications.

It is well known that studies on neural dynamical systems not only involve a discussion of stability properties, but also involve many dynamic behaviors such as periodic oscillation, bifurcation, and chaos. In many applications, the properties of periodic solutions are of great interest, which have been successfully applied in, for example, learning theory [21] since effective learning usually requires repetition. In addition, an equilibrium point can be viewed as a special periodic solution of neural networks with arbitrary period. In this sense, the analysis of periodic solutions of neural networks can be considered to be more general than that of

This work was supported in part by the National Natural Science Foundation of China under Grant 50608072, an International Joint Project sponsored by the Royal Society of the U.K. and the National Natural Science Foundation of China, and the Alexander von Humboldt Foundation of Germany.

${ }^{a}$ Department of Mathematics, Chongqing Jiaotong University, Chongqing 400074, China. Email: qiankunsong@163.com

${ }^{b}$ Department of Information Systems and Computing, Brunel University, Uxbridge, Middlesex, UB8 3PH, United Kingdom. Email: Zidong.Wang@brunel.ac.uk, Fax: ++44/1895 251686.

*Corresponding author. 
equilibrium point. Recently, the existence and stability of the periodic solution have been studied for recurrent neural networks with constant delays or time-varying delays, see e.g. [13, 19,27] and references therein.

It is worth noticing that, up to now, most recurrent neural networks have been assumed to act in a continuous-time manner. However, when implementing the continuous-time recurrent neural network for computer simulation, for experimental or computational purposes, it is essential to formulate a discrete-time system that is an analogue of the continuous-time recurrent neural network. To some extent, the discrete-time analogue inherits the dynamical characteristics of the continuous-time recurrent neural network under mild or no restriction on the discretization step-size, and also remains functional similarity to the continuous-time recurrent neural network and any physical or biological reality that the continuous-time recurrent neural network has [15]. Unfortunately, as pointed out in [16], the discretization can not preserve the dynamics of the continuous-time counterpart even for a small sampling period. Therefore, there is a crucial need to study the dynamics of discrete-time neural networks.

Recently, the dynamics analysis problem for discrete-time recurrent neural networks with or without time delays has received considerable research interest, see for example $[6,9,11,12,14-16,20,28-31]$ and references therein. In [12,14-16], the global exponential stability has been investigated for discrete-time delayed recurrent neural networks, and several sufficient conditions for checking the global exponential stability of the equilibrium point have been obtained. In [9], the global robust stability problem has been considered for a general class of discrete-time interval neural networks that contain time-invariant uncertain parameters with their values being unknown but bounded in given compact sets, and three sufficient conditions ensuring the global robust stability have been given. In [28,29], the authors have studied the stability and bifurcation problems for a class of discrete-time neural networks. In [31], the stability and convergence of the periodic solution have been studied for discrete-time neural network of two neurons. In [6,20], several sufficient conditions have been derived for checking the existence and global exponential stability of the periodic solution for discrete-time recurrent neural networks with constant delay. In [11], the existence of a unique almost periodic sequence solution has been studied for discrete-time neural networks without delay. In [30], the authors have investigated the existence and global exponential stability of the periodic solutions for discrete-time BAM neural networks with periodic coefficients and distributed delays,

It should be pointed out that, the given criteria in $[6,20]$ have been based upon certain diagonal dominance or $M$-matrix conditions on weight matrices of the networks, which only depend on absolute values of the weights and ignore the signs of the weights. Therefore, the conditions are somewhat conservative. In [14], a linear matrix inequality (LMI) approach has been developed to deal with the analysis problem of exponential stability for a class of discrete-time recurrent neural networks (DRNNs) with time delays. Unfortunately, the construction of Lyapunov functional used in [14] appeared to be conservative, and there is much room to reduce the possible conservatism. Motivated by the above discussions, the objective of this Letter is to study the existence and stability of the periodic solution for discrete-time recurrent neural network with time-varying delays by employing a new Lyapunov-Krasovskii functional as well as a unified LMI approach. Under more general description on the activation functions, we utilize the latest free-weighting matrix method $[3-5,7,8]$ and obtain several less conservative conditions, which can be checked numerically using the effective LMI toolbox in MATLAB. Two simulation examples are given to show the effectiveness and less conservatism of the proposed criteria.

Notations: The notations are quite standard. Throughout this Letter, $R^{n}$ and $R^{n \times m}$ denote, respectively, the $n$-dimensional Euclidean space and the set of all $n \times m$ real matrices. The superscript " $T$ " denotes matrix 
transposition. The notation $X \geq Y$ (respectively, $X>Y$ ) means that $X$ and $Y$ are symmetric matrices, and that $X-Y$ is positive semidefinite (respectively, positive definite). $\|\cdot\|$ is the Euclidean norm in $R^{n}$. If $A$ is a matrix, denote by $\|A\|$ its operator norm, i.e., $\|A\|=\sup \{\|A x\|:\|x\|=1\}=\sqrt{\lambda_{\max }\left(A^{T} A\right)}$, where $\lambda_{\max }(A)$ (respectively, $\lambda_{\min }(A)$ ) means the largest (respectively, smallest) eigenvalue of $A$. For integers $a, b$, and $a<b$, $N[a, b]$ denotes the discrete interval given $N[a, b]=\{a, a+1, \cdots, b-1, b\} . C\left(N[-\tau, 0], R^{n}\right)$ denotes the set of all functions $\phi: N[-\tau, 0] \rightarrow R^{n}$. Sometimes, the arguments of a function or a matrix will be omitted in the analysis when no confusion can arise.

\section{MOdEL DESCRIPTION AND PRELIMINARIES}

In this Letter, we consider the following neural network model

$$
x(k+1)=C x(k)+A f(x(k))+B f(x(k-\tau(k)))+I(k)
$$

for $k=1,2, \cdots$, where $x(k)=\left(x_{1}(k), x_{2}(k), \cdots, x_{n}(k)\right)^{T} \in R^{n}, x_{i}(k)$ is the state of the $i$ th neuron at time $k ; f(x(k))=\left(f_{1}\left(x_{1}(k)\right), f_{2}\left(x_{2}(k)\right), \cdots, f_{n}\left(x_{n}(k)\right)\right)^{T} \in R^{n}, f_{j}\left(x_{j}(k)\right)$ denotes the activation function of the $j$ th neuron at time $k ; I(k)=\left(I_{1}(k), I_{2}(k), \cdots, I_{n}(k)\right)^{T} \in R^{n}, I_{i}(k)$ represents the external input on the $i$ th neuron at time $k$; the positive integer $\tau(k)$ corresponds to the transmission delay and satisfies $\tau \leq \tau(k) \leq \bar{\tau}$ ( $\tau \geq 0$ and $\bar{\tau} \geq 0$ are known integers); $C=\operatorname{diag}\left(c_{1}, c_{2}, \cdots, c_{n}\right), c_{i}\left(0 \leq c_{i}<1\right)$ describes the rate with which the $i$ th neuron will reset its potential to the resting state in isolation when disconnected from the networks and external inputs; $A=\left(a_{i j}\right)_{n \times n}$ is the connection weight matrix, $B=\left(b_{i j}\right)_{n \times n}$ is the delayed connection weight matrix.

When $I(k)=I$ is constant, model (1) becomes the following model:

$$
x(k+1)=C x(k)+A f(x(k))+B f(x(k-\tau(k)))+I
$$

for $k=1,2, \cdots$.

The initial conditions associated with models (1) and (2) are given by

$$
x_{i}(s)=\phi_{i}(s), \quad s \in N[-\bar{\tau}, 0], \quad i=1,2, \cdots, n .
$$

Throughout this Letter, we make the following assumptions:

(H1) $I(k)$ and $\tau(k)$ are periodic functions with common period $\omega$ for $k=1,2, \cdots$.

(H2) There exist constants $F_{j}^{-}$and $F_{j}^{+}(i=1,2, \cdots, n)$ such that

$$
F_{j}^{-} \leq \frac{f_{j}\left(\alpha_{1}\right)-f_{j}\left(\alpha_{2}\right)}{\alpha_{1}-\alpha_{2}} \leq F_{j}^{+}
$$

for all $\alpha_{1} \neq \alpha_{2}$.

Definition 1: The periodic solution $x^{*}(k)$ of model (1) with (3) is said to be globally exponentially stable if there exist two positive constants $M>0$ and $0<\varepsilon<1$ such that

$$
\left\|x(k)-x^{*}(k)\right\| \leq M \varepsilon^{k} \sup _{s \in N[-\bar{\tau}, 0]}\left\|\phi(s)-\phi^{*}(s)\right\|
$$

for all $k=1,2, \cdots$, where $x(k)$ is any solution of model (1) with $(3), \phi(s)$ and $\phi^{*}(s)$ are the initial functions of solutions $x(k)$ and $x^{*}(k)$, respectively. 
Definition 2: The equilibrium point $x^{*}$ of model (2) with (3) is said to be globally exponentially stable if there exist two positive constants $M>0$ and $0<\varepsilon<1$ such that

$$
\left\|x(k)-x^{*}\right\| \leq M \varepsilon^{k} \sup _{s \in N[-\bar{\tau}, 0]}\left\|\phi(s)-x^{*}\right\|
$$

for all $k=1,2, \cdots$, where $x(k)$ is any solution of model $(2)$ with $(3), \phi(s)$ is the initial functions of solutions $x(k)$.

Lemma 1: [22] Given constant matrices $P, Q$ and $R$, where $P^{T}=P, Q^{T}=Q$, then

$$
\left[\begin{array}{cc}
P & R \\
R^{T} & -Q
\end{array}\right]<0
$$

is equivalent to the following conditions

$$
\begin{gathered}
Q>0, \quad P+R Q^{-1} R^{T}<0 . \\
\text { III. MAIN RESULT }
\end{gathered}
$$

In this section, we shall establish our main criteria based on the LMI approach.

For presentation convenience, in the following, we denote

$$
F_{1}=\operatorname{diag}\left(F_{1}^{-} F_{1}^{+}, F_{2}^{-} F_{2}^{+}, \cdots, F_{n}^{-} F_{n}^{+}\right), \quad F_{2}=\operatorname{diag}\left(\frac{F_{1}^{-}+F_{1}^{+}}{2}, \frac{F_{2}^{-}+F_{2}^{+}}{2}, \cdots, \frac{F_{n}^{-}+F_{n}^{+}}{2}\right) .
$$

Theorem 1: Under assumptions $(\mathbf{H 1})$ and $(\mathbf{H 2})$, there exists exactly one $\omega$-periodic solution of model (1) with (3) and all other solutions of model (1) with (3) converge exponentially to it as $k \rightarrow+\infty$, if there exist five $n \times n$ symmetric positive definite matrices $P, Q, R, Z_{1}$ and $Z_{2}$, two $n \times n$ positive diagonal matrices $D$ and $H$, three $5 n \times n$ matrices $S_{1}, S_{2}$ and $S_{3}$ such that the following LMI holds:

$$
W=\left[\begin{array}{cc}
W_{1}+\Xi+\Xi^{T} & W_{2} \\
W_{2}^{T} & -W_{3}
\end{array}\right]<0,
$$

where

$$
\begin{gathered}
W_{1}=\left[\begin{array}{ccccc}
\Pi_{1}-F_{1} D & 0 & 0 & \Pi_{2}+F_{2} D & \Pi_{3} \\
0 & -Q-F_{1} H & 0 & 0 & F_{2} H \\
0 & 0 & -R & 0 & 0 \\
\Pi_{2}^{T}+F_{2} D & 0 & 0 & \Pi_{4}-D & \Pi_{5} \\
\Pi_{3}^{T} & F_{2} H & 0 & \Pi_{5}^{T} & \Pi_{6}-H
\end{array}\right], \\
\Xi=\left[\begin{array}{lllll}
S_{1}+S_{3} & -S_{1}+S_{2} & -S_{2}-S_{3} & 0 & 0
\end{array}\right], \\
W_{2}=\left[\begin{array}{ccc}
\sqrt{\bar{\tau}} S_{1} & \sqrt{\bar{\tau}-\tau} S_{2} & \sqrt{\bar{\tau}} S_{3}
\end{array}\right], \quad W_{3}=\operatorname{diag}\left(Z_{1}, Z_{1}, Z_{2}\right),
\end{gathered}
$$

with

$$
\begin{gathered}
\Pi_{1}=C P C-P+(1+\bar{\tau}-\tau) Q+R+\bar{\tau}(C-E)\left(Z_{1}+Z_{2}\right)(C-E), \quad \Pi_{2}=C P A+\bar{\tau}(C-E)\left(Z_{1}+Z_{2}\right) A, \\
\Pi_{3}=C P B+\bar{\tau}(C-E)\left(Z_{1}+Z_{2}\right) B, \quad \Pi_{4}=A^{T} P A+\bar{\tau} A^{T}\left(Z_{1}+Z_{2}\right) A, \\
\Pi_{5}=A^{T} P B+\bar{\tau} A^{T}\left(Z_{1}+Z_{2}\right) B, \quad \Pi_{6}=B^{T} P B+\bar{\tau} B^{T}\left(Z_{1}+Z_{2}\right) B .
\end{gathered}
$$


Proof: $\quad$ Let $\Omega=\left\{\phi \mid \phi \in C\left(N[-\bar{\tau}, 0], R^{n}\right)\right\}$. For $\phi \in \Omega$, define

$$
\|\phi\|=\sup _{s \in N[-\bar{\tau}, 0]}\|\phi(s)\|,
$$

then $\Omega$ is a Banach space with the topology of uniform convergence.

For any $\phi, \psi \in \Omega$, let $x(k, \phi)$ and $x(k, \psi)$ be the solutions of model (1) starting from $\phi$ and $\psi$, respectively. Define

$$
x^{(k)}(\phi)(\theta)=x(k+\theta, \phi), \quad \theta \in N[-\bar{\tau}, 0], \quad k=1,2, \cdots,
$$

and then $x^{(k)}(\phi)(\theta) \in \Omega$ for all $k=1,2, \cdots$. It follows from model (1) that

$$
\begin{aligned}
x(k+1, \phi)-x(k+1, \psi)= & C(x(k, \phi)-x(k, \psi))+A(f(x(k, \phi))-f(x(k, \psi))) \\
& +B(f(x(k-\tau(k), \phi))-f(x(k-\tau(k), \psi))) .
\end{aligned}
$$

Letting

$$
y(k)=x(k, \phi)-x(k, \psi), \quad g(k)=f(x(k, \phi))-f(x(k, \psi)),
$$

model (6) can then be simplified as

$$
y(k+1)=C y(k)+A g(k)+B g(k-\tau(k)) .
$$

Consider the following Lyapunov-Krasovskii functional candidate for model (7) as

$$
V(k)=V_{1}(k)+V_{2}(k)+V_{3}(k)+V_{4}(k)+V_{5}(k),
$$

where

$$
\begin{gathered}
V_{1}(k)=y^{T}(k) P y(k), \\
V_{2}(k)=\sum_{i=k-\tau(k)}^{k-1} y^{T}(i) Q y(i), \\
V_{3}(k)=\sum_{l=k-\bar{\tau}+1}^{k-\tau} \sum_{i=l}^{k-1} y^{T}(i) Q y(i), \\
V_{4}(k)=\sum_{i=k-\bar{\tau}}^{k-1} y^{T}(i) R y(i), \\
V_{5}(k)=\sum_{i=-\bar{\tau}} \sum_{l=k+i}^{k-1} \eta^{T}(l)\left(Z_{1}+Z_{2}\right) \eta(l), \\
\eta(k)=y(k+1)-y(k) .
\end{gathered}
$$


Calculating the difference of $V_{i}(k)(i=1,2, \cdots, 5)$ along the trajectories of model $(7)$, we obtain

$$
\begin{aligned}
& \Delta V_{1}(k)=(C y(k)+A g(k)+B g(k-\tau(k)))^{T} P(C y(k)+A g(k)+B g(k-\tau(k)))-y^{T}(k) P y(k) \\
& =y^{T}(k)(C P C-P) y(k)+2 y^{T}(k) C P A g(k)+2 y^{T}(k) C P B g(k-\tau(k)) \\
& +g^{T}(k) A^{T} P A g(k)+2 g^{T}(k) A^{T} P B g(k-\tau(k))+g^{T}(k-\tau(k)) B^{T} P B g(k-\tau(k)), \\
& \Delta V_{2}(k)=\sum_{i=k+1-\tau(k+1)}^{k} y^{T}(i) Q y(i)-\sum_{i=k-\tau(k)}^{k-1} y^{T}(i) Q y(i) \\
& =\sum_{i=k+1-\tau(k+1)}^{k-\tau} y^{T}(i) Q y(i)+\sum_{i=k-\tau+1}^{k-1} y^{T}(i) Q y(i)+y^{T}(k) Q y(k) \\
& -\sum_{i=k-\tau(k)+1}^{k-1} y^{T}(i) Q y(i)-y^{T}(k-\tau(k)) Q y(k-\tau(k)) \\
& \leq \sum_{i=k+1-\tau(k+1)}^{k-\tau} y^{T}(i) Q y(i)+y^{T}(k) Q y(k)-y^{T}(k-\tau(k)) Q y(k-\tau(k)) \\
& \leq \sum_{i=k+1-\bar{\tau}}^{k-\tau} y^{T}(i) Q y(i)+y^{T}(k) Q y(k)-y^{T}(k-\tau(k)) Q y(k-\tau(k)), \\
& \Delta V_{3}(k)=\sum_{l=k-\bar{\tau}+2}^{k+1-\tau} \sum_{i=l}^{k} y^{T}(i) Q y(i)-\sum_{l=k-\bar{\tau}+1}^{k-\tau} \sum_{i=l}^{k-1} y^{T}(i) Q y(i) \\
& =(\bar{\tau}-\tau) y^{T}(k) Q y(k)-\sum_{l=k-\bar{\tau}+1}^{k-\tau} y^{T}(l) Q y(l), \\
& \Delta V_{4}(k)=y^{T}(k) R y(k)-y^{T}(k-\bar{\tau}) R y(k-\bar{\tau}),
\end{aligned}
$$

and

$$
\begin{aligned}
\Delta V_{5}(k)= & \sum_{i=-\bar{\tau}}^{-1}\left[\eta^{T}(k)\left(Z_{1}+Z_{2}\right) \eta(k)-\eta^{T}(k+i)\left(Z_{1}+Z_{2}\right) \eta(k+i)\right] \\
= & \bar{\tau} \eta^{T}(k)\left(Z_{1}+Z_{2}\right) \eta(k)-\sum_{i=k-\bar{\tau}}^{k-\tau(k)-1} \eta^{T}(i) Z_{1} \eta(i)-\sum_{i=k-\tau(k)}^{k-1} \eta^{T}(i) Z_{1} \eta(i)-\sum_{i=k-\bar{\tau}}^{k-1} \eta^{T}(i) Z_{2} \eta(i) \\
= & \bar{\tau}\left[y^{T}(k)(C-E)\left(Z_{1}+Z_{2}\right)(C-E) y(k)+2 y^{T}(k)(C-E)\left(Z_{1}+Z_{2}\right) A g(k)\right. \\
& +2 y^{T}(k)(C-E)\left(Z_{1}+Z_{2}\right) B g(k-\tau(k))+g^{T}(k) A^{T}\left(Z_{1}+Z_{2}\right) A g(k) \\
& \left.+2 g^{T}(k) A^{T}\left(Z_{1}+Z_{2}\right) B g(k-\tau(k))+g^{T}(k-\tau(k)) B^{T}\left(Z_{1}+Z_{2}\right) B g(k-\tau(k))\right] \\
& \quad-\sum_{i=k-\bar{\tau}}^{k-\tau(k)-1} \eta^{T}(i) Z_{1} \eta(i)-\sum_{i=k-\tau(k)}^{k-1} \eta^{T}(i) Z_{1} \eta(i)-\sum_{i=k-\bar{\tau}}^{k-1} \eta^{T}(i) Z_{2} \eta(i) .
\end{aligned}
$$

Denoting

$$
\alpha(k)=\left(y^{T}(k), y^{T}(k-\tau(k)), y^{T}(k-\bar{\tau}), g^{T}(k), g^{T}(k-\tau(k))\right)^{T},
$$


it follows from (8), (14)-(18) that

$$
\Pi=\left[\begin{array}{ccccc}
\Pi_{1} & 0 & 0 & \Pi_{2} & \Pi_{3} \\
0 & -Q & 0 & 0 & 0 \\
0 & 0 & -R & 0 & 0 \\
\Pi_{2}^{T} & 0 & 0 & \Pi_{4} & \Pi_{5} \\
\Pi_{3}^{T} & 0 & 0 & \Pi_{5}^{T} & \Pi_{6}
\end{array}\right]
$$

$$
\begin{aligned}
& \Delta V(k) \leq \alpha^{T}(k) \Pi \alpha(k)-\sum_{i=k-\bar{\tau}}^{k-\tau(k)-1} \eta^{T}(i) Z_{1} \eta(i)-\sum_{i=k-\tau(k)}^{k-1} \eta^{T}(i) Z_{1} \eta(i)-\sum_{i=k-\bar{\tau}}^{k-1} \eta^{T}(i) Z_{2} \eta(i) \\
& =\alpha^{T}(k) \Pi \alpha(k)-\sum_{i=k-\bar{\tau}}^{k-\tau(k)-1} \eta^{T}(i) Z_{1} \eta(i)-\sum_{i=k-\tau(k)}^{k-1} \eta^{T}(i) Z_{1} \eta(i)-\sum_{i=k-\bar{\tau}}^{k-1} \eta^{T}(i) Z_{2} \eta(i) \\
& +2 \alpha^{T}(k) S_{1}\left[y(k)-y(k-\tau(k))-\sum_{i=k-\tau(k)}^{k-1} \eta(i)\right] \\
& +2 \alpha^{T}(k) S_{2}\left[y(k-\tau(k))-y(k-\bar{\tau})-\sum_{i=k-\bar{\tau}}^{k-\tau(k)-1} \eta(i)\right] \\
& +2 \alpha^{T}(k) S_{3}\left[y(k)-y(k-\bar{\tau})-\sum_{i=k-\bar{\tau}}^{k-1} \eta(i)\right] \\
& \leq \alpha^{T}(k)\left(\Pi+\Xi+\Xi^{T}+\bar{\tau} S_{1} Z_{1}^{-1} S_{1}^{T}+(\bar{\tau}-\tau) S_{2} Z_{1}^{-1} S_{2}^{T}+\bar{\tau} S_{3} Z_{2}^{-1} S_{3}^{T}\right) \alpha(k) \\
& -\sum_{i=k-\tau(k)}^{k-1}\left(S_{1}^{T} \alpha(k)+Z_{1} \eta(i)\right)^{T} Z_{1}^{-1}\left(S_{1}^{T} \alpha(k)+Z_{1} \eta(i)\right) \\
& -\sum_{i=k-\bar{\tau}}^{k-\tau(k)-1}\left(S_{2}^{T} \alpha(k)+Z_{1} \eta(i)\right)^{T} Z_{1}^{-1}\left(S_{2}^{T} \alpha(k)+Z_{1} \eta(i)\right) \\
& -\sum_{i=k-\bar{\tau}}^{k-1}\left(S_{3}^{T} \alpha(k)+Z_{2} \eta(i)\right)^{T} Z_{2}^{-1}\left(S_{3}^{T} \alpha(k)+Z_{2} \eta(i)\right) \\
& \leq \alpha^{T}(k)\left(\Pi+\Xi+\Xi^{T}+\bar{\tau} S_{1} Z_{1}^{-1} S_{1}^{T}+(\bar{\tau}-\tau) S_{2} Z_{1}^{-1} S_{2}^{T}+\bar{\tau} S_{3} Z_{2}^{-1} S_{3}^{T}\right) \alpha(k) .
\end{aligned}
$$

From (H2), we have

$$
\begin{gathered}
\left(g_{i}(k)-F_{i}^{-} y_{i}(k)\right)\left(g_{i}(k)-F_{i}^{+} y_{i}(k)\right) \leq 0, \quad i=1,2, \cdots, n, \\
\left(g_{i}(k-\tau(k))-F_{i}^{-} y_{i}(k-\tau(k))\right)\left(g_{i}(k-\tau(k))-F_{i}^{+} y_{i}(k-\tau(k))\right) \leq 0, \quad i=1,2, \cdots, n,
\end{gathered}
$$

which are equivalent to

$$
\begin{gathered}
{\left[\begin{array}{c}
y(k) \\
g(k)
\end{array}\right]^{T}\left[\begin{array}{cc}
F_{j}^{-} F_{j}^{+} e_{j} e_{j}^{T} & -\frac{F_{j}^{-}+F_{j}^{+}}{2} e_{j} e_{j}^{T} \\
-\frac{F_{j}^{-}+F_{j}^{+}}{2} e_{j} e_{j}^{T} & e_{j} e_{j}^{T}
\end{array}\right]\left[\begin{array}{c}
y(k) \\
g(k)
\end{array}\right] \leq 0, \quad j=1,2, \cdots, m,} \\
{\left[\begin{array}{c}
y(k-\tau(k)) \\
g(k-\tau(k))
\end{array}\right]^{T}\left[\begin{array}{cc}
F_{j}^{-} F_{j}^{+} e_{j} e_{j}^{T} & -\frac{F_{j}^{-}+F_{j}^{+}}{2} e_{j} e_{j}^{T} \\
-\frac{F_{j}^{-}+F_{j}^{+}}{2} e_{j} e_{j}^{T} & e_{j} e_{j}^{T}
\end{array}\right]\left[\begin{array}{l}
y(k-\tau(k)) \\
g(k-\tau(k))
\end{array}\right] \leq 0, \quad j=1,2, \cdots, m,}
\end{gathered}
$$


where $e_{r}$ denotes the unit column vector having 1 element on its $r$ th row and zeros elsewhere.

Letting $D=\operatorname{diag}\left(d_{1}, d_{2}, \cdots, d_{n}\right)$ and $H=\operatorname{diag}\left(h_{1}, h_{2}, \cdots, h_{n}\right)$, we have from (19)-(21) that

$$
\begin{aligned}
\Delta V(k) \leq & \alpha^{T}(k)\left(\Pi+\Xi+\Xi^{T}+\bar{\tau} S_{1} Z_{1}^{-1} S_{1}^{T}+(\bar{\tau}-\tau) S_{2} Z_{1}^{-1} S_{2}^{T}+\bar{\tau} S_{3} Z_{2}^{-1} S_{3}^{T}\right) \alpha(k) \\
& -\sum_{j=1}^{m} d_{j}\left[\begin{array}{l}
y(k) \\
g(k)
\end{array}\right]^{T}\left[\begin{array}{cc}
F_{j}^{-} F_{j}^{+} e_{j} e_{j}^{T} & -\frac{F_{j}^{-}+F_{j}^{+}}{2} e_{j} e_{j}^{T} \\
-\frac{F_{j}^{-}+F_{j}^{+}}{2} e_{j} e_{j}^{T} & e_{j} e_{j}^{T}
\end{array}\right]\left[\begin{array}{c}
y(k) \\
g(k)
\end{array}\right] \\
& -\sum_{j=1}^{m} h_{j}\left[\begin{array}{l}
y(k-\tau(k)) \\
g(k-\tau(k))
\end{array}\right]^{T}\left[\begin{array}{cc}
F_{j}^{-} F_{j}^{+} e_{j} e_{j}^{T} & -\frac{F_{j}^{-}+F_{j}^{+}}{2} e_{j} e_{j}^{T} \\
-\frac{F_{j}^{-}+F_{j}^{+}}{2} e_{j} e_{j}^{T} & e_{j} e_{j}^{T}
\end{array}\right]\left[\begin{array}{c}
y(k-\tau(k)) \\
g(k-\tau(k))
\end{array}\right] \\
= & \alpha^{T}(k)\left(\Pi+\Xi+\Xi^{T}+\bar{\tau} S_{1} Z_{1}^{-1} S_{1}^{T}+(\bar{\tau}-\tau) S_{2} Z_{1}^{-1} S_{2}^{T}+\bar{\tau} S_{3} Z_{2}^{-1} S_{3}^{T}\right) \alpha(k) \\
& -\left[\begin{array}{l}
y(k) \\
g(k)
\end{array}\right]^{T}\left[\begin{array}{cc}
F_{1} D & -F_{2} D \\
-F_{2} D & D
\end{array}\right]\left[\begin{array}{l}
y(k) \\
g(k)
\end{array}\right] \\
& -\left[\begin{array}{ll}
y(k-\tau(k)) \\
g(k-\tau(k))
\end{array}\right]^{T}\left[\begin{array}{cc}
F_{1} H & -F_{2} H \\
-F_{2} H & H
\end{array}\right]\left[\begin{array}{l}
y(k-\tau(k)) \\
g(k-\tau(k))
\end{array}\right] \\
= & \alpha^{T}(k)\left(W_{1}+\Xi+\Xi^{T}+\bar{\tau} S_{1} Z_{1}^{-1} S_{1}^{T}+(\bar{\tau}-\tau) S_{2} Z_{1}^{-1} S_{2}^{T}+\bar{\tau} S_{3} Z_{2}^{-1} S_{3}^{T}\right) \alpha(k) .
\end{aligned}
$$

Define $G=W_{1}+\Xi+\Xi^{T}+\bar{\tau} S_{1} Z_{1}^{-1} S_{1}^{T}+(\bar{\tau}-\tau) S_{2} Z_{1}^{-1} S_{2}^{T}+\bar{\tau} S_{3} Z_{2}^{-1} S_{3}^{T}$. From (5) and Lemma 1, we know that $G<0$ and therefore

$$
\Delta V(k) \leq-\lambda_{\min }(-G)\|\alpha(k)\|^{2} \leq-\lambda_{\min }(-G)\|y(k)\|^{2} .
$$

From the definition of $V(k)$, it is easy to verify that

$$
V(k) \leq \lambda_{\max }(P)\|y(k)\|^{2}+\rho \sum_{i=k-\bar{\tau}}^{k-1}\|y(i)\|^{2}+2 \bar{\tau} \lambda_{\max }\left(Z_{1}+Z_{2}\right) \sum_{i=k-\bar{\tau}}^{k-1}\|y(i+1)\|^{2},
$$

where $\rho=(1+\bar{\tau}-\tau) \lambda_{\max }(Q)+\lambda_{\max }(R)+2 \bar{\tau} \lambda_{\max }\left(Z_{1}+Z_{2}\right)$.

For any scalar $\mu>1$, it follows from (22) and (23) that

$$
\begin{aligned}
\mu^{j+1} V(j+1)-\mu^{j} V(j)= & \mu^{j+1} \Delta V(j)+\mu^{j}(\mu-1) V(j) \\
\leq & {\left[\mu^{j}(\mu-1) \lambda_{\max }(P)-\mu^{j+1} \lambda_{\min }(-G)\right]\|y(j)\|^{2} } \\
& +\rho \mu^{j}(\mu-1) \sum_{i=j-\bar{\tau}}^{j-1}\|y(i)\|^{2} \\
& +2 \bar{\tau} \lambda_{\max }\left(Z_{1}+Z_{2}\right) \mu^{j}(\mu-1) \sum_{i=j-\bar{\tau}}^{j-1}\|y(i+1)\|^{2} .
\end{aligned}
$$

Summing up both sides of (24) from 0 to $k-1$ with respect to $j$, we have

$$
\begin{aligned}
\mu^{k} V(k)-V(0) \leq & {\left[(\mu-1) \lambda_{\max }(P)-\mu \lambda_{\min }(-G)\right] \sum_{j=0}^{k-1} \mu^{j}\|y(j)\|^{2} } \\
& +\rho(\mu-1) \sum_{j=0}^{k-1} \sum_{i=j-\bar{\tau}}^{j-1} \mu^{j}\|y(i)\|^{2} \\
& +2 \bar{\tau} \lambda_{\max }\left(Z_{1}+Z_{2}\right)(\mu-1) \sum_{j=0}^{k-1} \sum_{i=j-\bar{\tau}}^{j-1} \mu^{j}\|y(i+1)\|^{2} .
\end{aligned}
$$


It is easy to compute that

$$
\begin{aligned}
\sum_{j=0}^{k-1} \sum_{i=j-\bar{\tau}}^{j-1} \mu^{j}\|y(i)\|^{2} & \leq\left(\sum_{i=-\bar{\tau}}^{-1} \sum_{j=0}^{i+\bar{\tau}}+\sum_{i=0}^{k-1-\bar{\tau}} \sum_{j=i+1}^{i+\bar{\tau}}+\sum_{i=k-\bar{\tau}}^{k-1} \sum_{j=i+1}^{k-1}\right) \mu^{j}\|y(i)\|^{2} \\
& \leq \bar{\tau} \mu^{\bar{\tau}} \sup _{s \in N[-\bar{\tau}, 0]}\|y(s)\|^{2}+\bar{\tau} \mu^{\bar{\tau}} \sum_{i=0}^{k-1} \mu^{i}\|y(i)\|^{2} .
\end{aligned}
$$

Similarly, we have

$$
\sum_{j=0}^{k-1} \sum_{i=j-\bar{\tau}}^{j-1} \mu^{j}\|y(i+1)\|^{2} \leq \bar{\tau} \mu^{\bar{\tau}} \sup _{s \in N[-\bar{\tau}, 0]}\|y(s)\|^{2}+\bar{\tau} \mu^{\bar{\tau}} \sum_{i=1}^{k} \mu^{i}\|y(i)\|^{2} .
$$

From (23), we obtain

$$
V(0) \leq\left[\lambda_{\max }(P)+\rho \bar{\tau}+2 \bar{\tau}^{2} \lambda_{\max }\left(Z_{1}+Z_{2}\right)\right] \sup _{s \in N[-\bar{\tau}, 0]}\|y(s)\|^{2} .
$$

It follows from (25)-(28) that

$$
\mu^{k} V(k) \leq L_{1}(\mu) \sup _{s \in N[-\bar{\tau}, 0]}\|y(s)\|^{2}+L_{2}(\mu) \sum_{j=0}^{k} \mu^{i}\|y(i)\|^{2},
$$

where

$$
\begin{gathered}
L_{1}(\mu)=\lambda_{\max }(P)+\rho \bar{\tau}+2 \bar{\tau}^{2} \lambda_{\max }\left(Z_{1}+Z_{2}\right)+(\mu-1) \rho \bar{\tau} \mu^{\bar{\tau}}+2(\mu-1) \bar{\tau}^{2} \mu^{\bar{\tau}} \lambda_{\max }\left(Z_{1}+Z_{2}\right), \\
L_{2}(\mu)=(\mu-1) \lambda_{\max }(P)-\mu \lambda_{\min }(-G)+(\mu-1) \rho \bar{\tau} \mu^{\bar{\tau}}+2(\mu-1) \bar{\tau}^{2} \mu^{\bar{\tau}} \lambda_{\max }\left(Z_{1}+Z_{2}\right) .
\end{gathered}
$$

Since $L_{2}(1)<0$, by the continuity of functions $L_{2}(\mu)$, we can choose a scalar $\beta>1$ such that $L_{2}(\beta) \leq 0$. Obviously, $L_{1}(\beta)>0$. From (29), we get

$$
\beta^{k} V(k) \leq L_{1}(\beta) \sup _{s \in N[-\bar{\tau}, 0]}\|y(s)\|^{2} .
$$

From the definition of $V(k)$, we have

$$
V(k) \geq \lambda_{\min }(P)\|y(k)\|^{2} .
$$

Let $M=\sqrt{\frac{L_{1}(\beta)}{\lambda_{\min }(P)}}, \varepsilon=\sqrt{\frac{1}{\beta}}$, then $M>0,0<\varepsilon<1$. It follows from (30) and (31) that

$$
\|y(k)\| \leq M \varepsilon^{k} \sup _{s \in N[-\bar{\tau}, 0]}\|y(s)\|
$$

for all $k=1,2, \cdots$. That is

$$
\|x(k, \phi)-x(k, \psi)\| \leq M \varepsilon^{k}\|\phi-\psi\|
$$

for all $k=1,2, \cdots$.

We can choose a positive integer $L$ such that

$$
M \varepsilon^{L \omega} \leq \frac{1}{4}
$$

Define a Poincarè mapping $\theta: \Omega \rightarrow \Omega$ by

$$
\theta(\phi)=x^{(\omega)}(\phi)
$$


Then, we can derive from (32) and (33) that

$$
\left\|\theta^{(L)}(\phi)-\theta^{(L)}(\psi)\right\| \leq \frac{1}{4}\|\phi-\psi\|
$$

which implies that $\theta^{(L)}$ is a contraction mapping and therefore there exists a unique fixed point $\phi^{*} \in \Omega$ such that

$$
\theta^{(L)}\left(\phi^{*}\right)=\phi^{*}
$$

Note that

$$
\theta^{(L)}\left(\theta\left(\phi^{*}\right)\right)=\theta\left(\theta^{(L)}\left(\phi^{*}\right)\right)=\theta\left(\phi^{*}\right),
$$

which shows that $\theta\left(\phi^{*}\right) \in \Omega$ is also a fixed point of $\theta^{(L)}$, and hence

$$
\theta\left(\phi^{*}\right)=\phi^{*}
$$

or

$$
x^{(\omega)}\left(\phi^{*}\right)=\phi^{*}
$$

Let $x\left(k, \phi^{*}\right)$ be the solution of model (1) through $\left(0, \phi^{*}\right)$. From assumption $(\mathbf{H} 1)$, we know that $x\left(k+\omega, \phi^{*}\right)$ is also a solution of model (1). It follows from the fact

$$
x^{(k+\omega)}\left(\phi^{*}\right)=x^{(k)}\left(x^{(\omega)}\left(\phi^{*}\right)\right)=x^{(k)}\left(\phi^{*}\right)
$$

that

$$
x\left(k+\omega, \phi^{*}\right)=x\left(k, \phi^{*}\right)
$$

for $k=1,2, \cdots$, which indicates that $x\left(k, \phi^{*}\right)$ is exactly one $\omega$-periodic solution of model (1). To this end, it is easy to see that all other solutions of model (1) converge exponentially to it as $k \rightarrow+\infty$. The proof is completed.

Remark 1: In assumption (H2) of this Letter, the constants $F_{j}^{-}$and $F_{j}^{+}(i=1,2, \cdots, n)$ are allowed to be positive, negative or zero. Hence, assumption (H2) is weaker than the following assumption (H3):

(H3) There exists a positive diagonal matrix $F=\operatorname{diag}\left(F_{1}, F_{2}, \cdots, F_{n}\right)$ such that

$$
\left|f_{j}\left(u_{1}\right)-f_{j}\left(u_{2}\right)\right| \leq F_{j}\left|u_{1}-u_{2}\right|
$$

for all $u_{1}, u_{2} \in R, j=1,2, \cdots, n$. Assumption (H3) was mostly used in literature $[6,12,15,16,20,30]$. Obviously, the activation functions such as sigmoid type and piecewise linear type are also the special case of the function satisfying assumption (H2).

Remark 2: In $[6,20]$, the given periodicity criteria for discrete-time recurrent neural network with constant delays were based upon spectral radius or M-matrix conditions on weight matrices of the networks, which only depend on absolute values of the weights and ignore the signs of the weights, and hence are somewhat conservative.

Remark 3: In model (2), for any constant $T \geq 0$, we have $I=I(k+T)=I(k)$ for $k=1,2, \cdots$. Thus, by the above result, when the sufficient condition in Theorem 1 is satisfied, one unique periodic solution becomes a periodic solution with any positive constants as its period. So, the periodic solution reduced to a constant solution, that is, an equilibrium point. Furthermore, all other solutions globally exponentially converge to this equilibrium point as $k \rightarrow+\infty$. The unique equilibrium point of model (2) is globally exponentially stable. Thus, by applying Theorem 1, we can easily obtain the following result. 
Corollary 1: Under assumption (H2) and condition (5), model (2) has one unique equilibrium point, which is globally exponentially stable.

Remark 4: In $[12,14,30]$, the boundedness of activation functions was required. In [9], the differentiability of activation functions was required. However, the boundedness and differentiability of activation functions have been removed in this Letter. Moreover, as shown in $[3-5,7,8]$, the use of the latest free-weighting matrix method in this Letter leads to delay-dependent and less conservative LMI conditions, which can be checked numerically using the effective LMI toolbox in MATLAB.

\section{ExAmples}

Example 1. Consider a discrete-time recurrent neural network (1), where

$$
\begin{gathered}
C=\left[\begin{array}{cc}
0.1 & 0 \\
0 & 0.2
\end{array}\right], \quad A=\left[\begin{array}{cc}
-0.1 & 0.1 \\
-0.1 & 0.05
\end{array}\right], \quad B=\left[\begin{array}{cc}
0.05 & 0.1 \\
0.05 & 0.05
\end{array}\right], \\
f_{1}(y)=\tanh (2 y), f_{2}(y)=\tanh (-4 y), I_{1}(k)=-3 \sin \left(\frac{k \pi}{2}\right), I_{2}(k)=2 \cos \left(\frac{k \pi}{2}\right), \tau(k)=3+\sin \left(\frac{k \pi}{2}\right) .
\end{gathered}
$$

It can be verified that assumptions $(\mathbf{H 1})$ and $(\mathbf{H 2})$ are satisfied with $F_{1}^{-}=0, F_{1}^{+}=2, F_{2}^{-}=-4, F_{2}^{+}=0$, $\tau=2, \bar{\tau}=4, \omega=4$. Thus,

$$
F_{1}=\left[\begin{array}{ll}
0 & 0 \\
0 & 0
\end{array}\right], \quad F_{2}=\left[\begin{array}{cc}
1 & 0 \\
0 & -2
\end{array}\right] .
$$

By the Matlab LMI Control Toolbox, we find a solution to the LMI in (5) as follows:

$$
\begin{aligned}
& P=\left[\begin{array}{cc}
1055.1 & -888.9 \\
-888.9 & 2845.7
\end{array}\right], Q=\left[\begin{array}{cc}
129.6014 & -143.8212 \\
-143.8212 & 498.7914
\end{array}\right], R=\left[\begin{array}{cc}
55.2379 & -43.8092 \\
-43.8092 & 96.5058
\end{array}\right] \\
& Z_{1}=\left[\begin{array}{cc}
49.3081 & -68.1327 \\
-68.1327 & 110.0119
\end{array}\right], Z_{2}=\left[\begin{array}{cc}
39.2746 & -56.1519 \\
-56.1519 & 87.8631
\end{array}\right] \\
& D=\left[\begin{array}{cc}
129.4471 & 0 \\
0 & 66.6214
\end{array}\right], H=\left[\begin{array}{cc}
47.5456 & 0 \\
0 & 42.5023
\end{array}\right] \text {, } \\
& S_{1}=\left[\begin{array}{cc}
1.3014 & -2.0846 \\
-4.7929 & 7.9712 \\
7.5433 & -13.4121 \\
-33.3671 & 52.4395 \\
4.5495 & -4.3859 \\
-3.9036 & 8.3979 \\
-0.4771 & 0.7415 \\
-1.2612 & 2.0908 \\
-3.5770 & 4.0615 \\
-6.4967 & 11.4255
\end{array}\right], S_{2}=\left[\begin{array}{cc}
1.3014 & -2.0846 \\
-4.7929 & 7.9712 \\
7.5433 & -13.4121 \\
-33.3671 & 52.4395 \\
4.5495 & -4.3859 \\
-3.9036 & 8.3979 \\
-0.4771 & 0.7415 \\
-1.2612 & 2.0908 \\
-3.5770 & 4.0615 \\
-6.4967 & 11.4255
\end{array}\right], S_{3}=\left[\begin{array}{cc}
-0.3899 & 1.9731 \\
-11.8190 & 19.5288 \\
0.5649 & -0.8447 \\
-0.3821 & 0.7001 \\
1.0156 & -0.9129 \\
-0.6661 & 1.5394 \\
-2.9507 & 3.4808 \\
-5.8679 & 9.4122 \\
-0.9554 & 1.4179 \\
1.0456 & -1.5582
\end{array}\right],
\end{aligned}
$$

Therefore, by Theorem 1, we know that model (1) with above given parameters has exactly one 4-periodic solution and all other solutions of the model converge exponentially to it as $k \rightarrow+\infty$, which is further verified by the simulation given in Figure 1. 

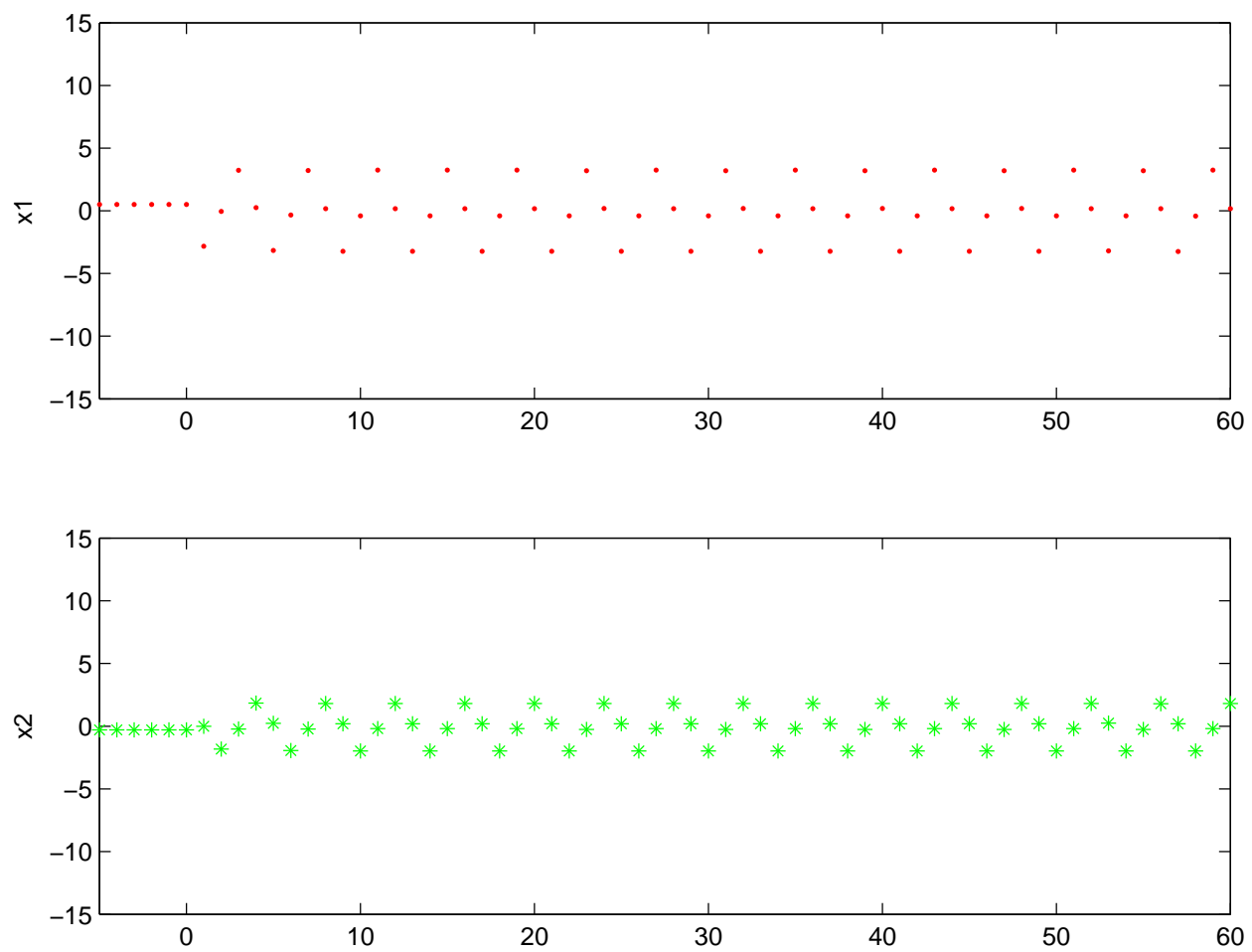

Fig. 1. State responses of the discrete-time recurrent neural network with initial conditions $\left(x_{1}(s), x_{2}(s)\right)^{T}=(0.5,-0.3)^{T}$, $s \in N[-4,0]$.

It should be pointed out that the theorems in $[6,20]$ are not applicable to judge the periodicity of this model since the criteria in $[6,20]$ fail.

Example 2. Consider a discrete-time recurrent neural network (2), where

$$
\begin{gathered}
C=\left[\begin{array}{cc}
0.26 & 0 \\
0 & 0.1
\end{array}\right], \quad A=\left[\begin{array}{cc}
0.1 & 0.2 \\
-0.15 & 0.1
\end{array}\right], \quad B=\left[\begin{array}{cc}
-0.25 & 0.1 \\
0.02 & 0.08
\end{array}\right], \\
f_{1}(y)=\frac{1}{2}(|y+1|+|y-1|), f_{2}(y)=|y+1|+|y-1|, I_{1}=-0.91, I_{2}=1.75, \tau(k)=3-\sin \left(\frac{k \pi}{2}\right) .
\end{gathered}
$$

It can be verified that assumption $(\mathbf{H 2})$ is satisfied with $F_{1}^{-}=-1, F_{1}^{+}=1, F_{2}^{-}=-2, F_{2}^{+}=2, \tau=2$, $\bar{\tau}=4$. Thus,

$$
F_{1}=\left[\begin{array}{cc}
-1 & 0 \\
0 & -4
\end{array}\right], \quad F_{2}=\left[\begin{array}{ll}
0 & 0 \\
0 & 0
\end{array}\right]
$$

By the Matlab LMI Control Toolbox, we find a solution to the LMI in (5) as follows:

$$
\begin{gathered}
P=\left[\begin{array}{cc}
422.9 & -15.4 \\
-15.4 & 1366.8
\end{array}\right], Q=\left[\begin{array}{cc}
69.8245 & -2.7674 \\
-2.7674 & 251.9583
\end{array}\right], R=\left[\begin{array}{cc}
19.0205 & -1.1514 \\
-1.1514 & 28.1610
\end{array}\right], \\
Z_{1}=\left[\begin{array}{cc}
19.6348 & -0.6881 \\
-0.6881 & 23.5347
\end{array}\right], Z_{2}=\left[\begin{array}{cc}
13.2610 & -1.0395 \\
-1.0395 & 19.6730
\end{array}\right], \\
D=\left[\begin{array}{cc}
74.6901 & 0 \\
0 & 100.0409
\end{array}\right], H=\left[\begin{array}{cc}
67.5113 & 0 \\
0 & 56.7165
\end{array}\right],
\end{gathered}
$$




$$
S_{1}=\left[\begin{array}{cc}
-0.7941 & -0.0682 \\
-0.0791 & 0.0065 \\
2.3542 & 0.2302 \\
0.2463 & 0.3708 \\
0.4211 & 0.0421 \\
0.0435 & 0.0739 \\
-0.1349 & -0.4254 \\
-0.3359 & 0.1254 \\
0.3963 & 0.1970 \\
-0.1724 & 0.1355
\end{array}\right], S_{2}=\left[\begin{array}{cc}
-0.3023 & -0.0292 \\
-0.0287 & -0.0506 \\
-4.1587 & -0.4056 \\
-0.4310 & -0.6434 \\
0.9700 & 0.1096 \\
0.1221 & 0.0737 \\
0.0015 & -0.0096 \\
0.0020 & 0.0042 \\
-0.0042 & 0.0035 \\
0.0010 & 0.0043
\end{array}\right], S_{3}=\left[\begin{array}{cc}
-0.6910 & -0.0533 \\
-0.0679 & -0.0141 \\
0.1991 & 0.0180 \\
0.0212 & 0.0403 \\
0.6629 & 0.0658 \\
0.0744 & 0.0905 \\
-0.0904 & -0.3559 \\
-0.2538 & 0.1109 \\
0.2994 & 0.1607 \\
-0.1343 & 0.1169
\end{array}\right]
$$

Therefore, by Corollary 1, we know that model (2) with above given parameters has one unique equilibrium point, which is globally exponentially stable. In fact, we can verify that $(1,3)^{T}$ is a unique equilibrium point of the model. The global exponential stability of equilibrium point $(1,3)^{T}$ is further verified by the simulation given in Figure 2.

It should be pointed out that the theorem in [14] are not applicable to judge the stability of this model since the activation functions are not bounded.
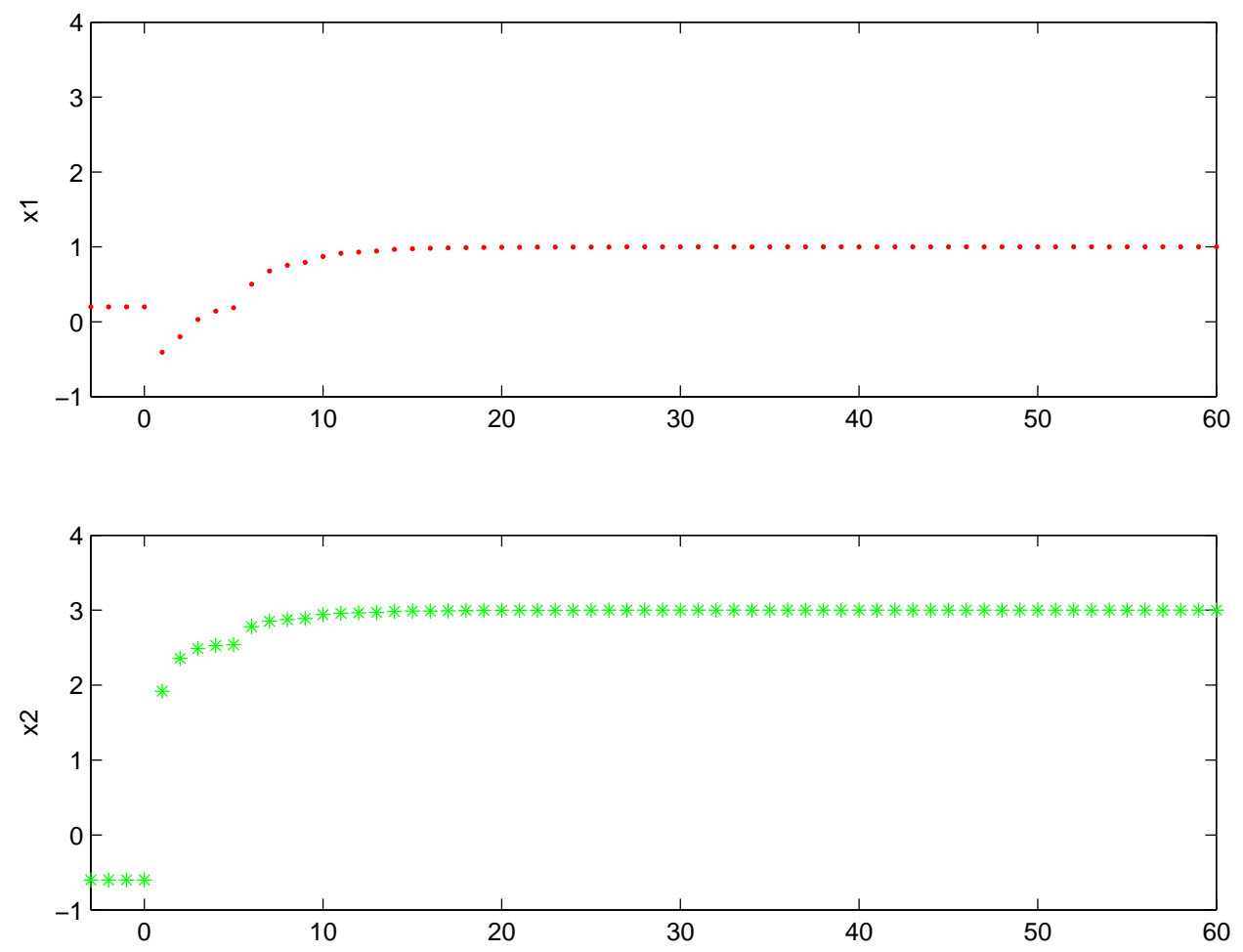

Fig. 2. State responses of the discrete-time recurrent neural network with initial conditions $\left(x_{1}(s), x_{2}(s)\right)^{T}=(0.2,-0.6)^{T}$, $s \in N[-4,0]$.

\section{Conclusions}

In this Letter, the existence and stability of periodic solution have been investigated for discrete-time recurrent neural network with time-varying delays. The description of the activation functions was more general than the recently commonly used Lipschitz conditions. By employing appropriate Lyapunov-Krasovskii func- 
tional and the free-weighting matrix method, a new LMI criterion is established to ensure the existence, uniqueness, and global exponential stability of periodic solution for the addressed neural network. The proposed results are less conservative than some recently known ones in the literature, which are demonstrated via two simulation examples.

We would like to point out that it is possible to generalize our main results to more complex neural networks, such as neural networks with parameter uncertainties (e.g. norm-bounded uncertainties [23, 25]), stochastic perturbations $[23,25,26]$, and Markovian jumping parameters [24]. The corresponding results will appear in the near future.

\section{REFERENCES}

[1] S. Arik, An analysis of exponential stability of delayed neural networks with time varying delays, Neural Networks, 17(7) (2004): 1027-1031.

[2] J. Cao, K. Yuan, H.X. Li, Global asymptotical stability of recurrent neural networks with multiple discrete delays and distributed delays. IEEE Transactions on Neural Networks, 17(6) (2006): 1646-1651.

[3] H. Gao and T. Chen, New results on stability of discrete-time systems with time-varying state delay, IEEE Transactions on Automatic Control, 52(2) (2007): 328-334.

[4] H. Gao, J. Lam, G. Chen, New criteria for synchronization stability of general complex dynamical networks with coupling delays, Physics Letters A, 360(2) (2006): 263-273.

[5] H. Gao, C. Wang, A delay-dependent approach to robust $H_{\infty}$ filtering for uncertain discrete-time state-delayed systems, IEEE Transactions on Signal Processing, 52(6) (2004): 1631-1640.

[6] S. Guo, L. Huang, Periodic oscillation for discrete-time Hopfield neural networks, Physics Letters A, 329(3) (2004): 199-206.

[7] Y. He, M. Wu and J.H. She, Delay-dependent exponential stability of delayed neural networks with time-varying delay, IEEE Transactions on Circuits and Systems II - Express Briefs, 53(7) (2006): 553-557.

[8] Y. He, M. Wu and J.H. She, An improved global asymptotic stability criterion for delayed cellular neural networks, IEEE Transactions on Neural Networks, 17(1) (2006): 250-252.

[9] S. Hu, J. Wang, Global robust stability of a class of discrete-time interval neural networks, IEEE Transactions on Circuits and Systems I, 53(1) (2006): 129-138.

[10] T. Huang, J. Cao, C. Li, Necessary and sufficient condition for the absolute exponential stability of a class of neural networks with finite delay, Physics Letters A, 352(1-2) (2006): 94-98.

[11] Z. Huang, X. Wang, F. Gao, The existence and global attractivity of almost periodic sequence solution of discrete-time neural networks, Physics Letters A, 350(3-4) (2006):182-191.

[12] J. Liang, J. Cao, D.W.C. Ho, Discrete-time bidirectional associative memory neural networks with variable delays, Physics Letters A, 335(2-3) (2005): 226-234.

[13] B. Liu, L. Huang, Existence of periodic solutions for cellular neural networks with complex deviating arguments, Applied Mathematics Letters, 20(1)(2007): 103-109.

[14] Y. Liu, Z. Wang, A. Serrano, X. Liu, Discrete-time recurrent neural networks with time-varying delays: Exponential stability analysis, Physics Letters A, 362(5-6) (2006): 480-488.

[15] S. Mohamad, Global exponential stability in continuous-time and discrete-time delayed bidirectional neural networks, Physica D, 159(3-4) (2001) 233-251.

[16] S. Mohamad, K. Gopalsamy, Exponential stability of continuous-time and discrete-time cellular neural networks with delays, Applied Mathematics and Computation, 135(1) (2003): 17-38.

[17] J.H. Park, A novel criterion for global asymptotic stability of BAM neural networks with time delays, Chaos, Solitons and Fractals, 29(2) (2006): 446-453.

[18] V. Singh, Simplified LMI condition for global asymptotic stability of delayed neural networks, Chaos, Solitons and Fractals, 29(2) (2006): 470-473.

[19] Q. Song, J. Cao, Dynamics of bidirectional associative memory networks with distributed delays and reaction-diffusion terms, Nonlinear Analysis: Real World Applications, 8(1)(2007):345-361.

[20] C. Sun, C. Feng, Exponential periodicity of continuous-time and discrete-time neural networks with delays, Neural Processing Letters, 19 (2004): 131-146. 
[21] S. Townley, A. Ilchmann, M.G. Weiss, W. Mcclements, A.C. Ruiz, D.H. Owens, D. Pratzel-Wolters, Existence and learning of oscillations in recurrent neural networks, IEEE Transactions on Neural Networks, 11(1) (2000): 205-214.

[22] Z. Wang, Y. Liu, X. Liu, On global asymptotic stability of neural networks with discrete and distributed delays, Physics Letters A, 345(4-6) (2005): 299-308.

[23] Z. Wang, S. Lauria, J. Fang, X. Liu, Exponential stability of uncertain stochastic neural networks with mixed time delays, Chaos, Solitons and Fractals, 32(1) (2007): 62-72.

[24] Z. Wang, Y. Liu, L. Yu, X. Liu, Exponential stability of delayed recurrent neural networks with Markovian jumping parameters, Physics Letters A, 356(4-5) (2006): 346-352.

[25] Z. Wang, Y. Liu, K. Fraser and X. Liu, Stochastic stability of uncertain Hopfield neural networks with discrete and distributed delays, Physics Letters A, 354(4) (2006): 288-297.

[26] Z. Wang, Y. Liu, M. Li and X. Liu, Stability analysis for stochastic Cohen-Grossberg neural networks with mixed time delays, IEEE Transactions on Neural Networks, 17(3), (2006): 814-820.

[27] Z. Yang, D. Xu, Existence and exponential stability of periodic solution for impulsive delay differential equations and applications, Nonlinear Analysis, 64(1)(2006): 130-145.

[28] Z. Yuan, D. Hu, L. Huang, Stability and bifurcation analysis on a discrete-time neural network, Journal of Computational and Applied Mathematics, 177(1) (2005): 89-100.

[29] H. Zhao, L. Wang, Stability and bifurcation for discrete-time Cohen-Grossberg neural network, Applied Mathematics and Computation, 179(2) (2006): 787-798.

[30] T. Zhou, Y. Liu, Y. Liu, Existence and global exponential stability of periodic solution for discrete-time BAM neural networks, Applied Mathematics and Computation, 182(2) (2006): 1341-1354.

[31] Z. Zhou, J.H. Wu, Stable periodic orbits in nonlinear discrete-time neural networks with delayed feedback, Computers and Mathematics with Applications, 45(6-9) (2003): 935-942. 